\title{
Dural Plasmacytoma Involving Calvarium with Soft Tissue Extension Mimicking Meningioma: A Diagnostic Dilemma
}

\author{
Anju Shukla ${ }^{1}$ Devendra Kumar Chhabra ${ }^{2}$ Tarun Kumar Pandey ${ }^{2}$ Prashant Raj Singh ${ }^{2}$ \\ ${ }^{1}$ Department of Lab Medicine, Sahara Hospital, Lucknow, \\ Uttar Pradesh, India \\ 2Department of Neurosurgery, Vivekananda Polyclinic and Institute \\ of Medical Sciences, Lucknow, Uttar Pradesh, India \\ Address for correspondence Anju Shukla, MD, \\ Department of Lab Medicine, Sahara Hospital, Viraj Khand, \\ Gomti Nagar, Lucknow 226010, Uttar Pradesh, India \\ (e-mail: anjushukla2012@gmail.com).
}

\begin{abstract}
Here, the authors describe a case of 25-year-old man diagnosed with dural plasmacytoma involving calvarium with soft tissue extension. Magnetic resonance imaging (MRI) revealed extra-axial heterogeneously enhancing soft tissue mass lesion in the left parieto-occipital region with a dural tail mimicking meningioma, destroying the left parietal bone, and bulging into the scalp. Mass was excised and histopathologic examination revealed plasmacytoma with amyloid deposition. There

Keywords

- dural plasmacytoma

- calvarium

- meningioma is no recurrence after postoperative radiotherapy and 2 years of follow-up, although the future course is still not certain. The clinician should consider alternative diagnosis other than meningioma prior to proceeding to surgery if the dural-based lesion is involving calvarium and soft tissue extension.
\end{abstract}

\section{Introduction}

Plasma cell tumors are characterized by monoclonal proliferation of plasma cells. They may appear as single lesions (solitary plasmacytoma) or multiple ones (multiple myeloma [MM]). Plasmacytomas usually develop in the bone, although they may also do so in soft tissues (called extramedullary plasmacytoma). ${ }^{1}$

Primary extramedullary plasmacytomas are uncommon tumors, mainly arising in the head and neck region, particularly in the upper aerodigestive tract, and represent approximately 1 to $4 \%$ of plasma cell tumors. ${ }^{2}$

Cranial plasmacytoma may arise from skull, leptomeninges, or the brain parenchyma. Meningeal involvement is a rare presentation, and only a few case reports have been described in the literature. These cases can easily be mistaken for meningioma, metastasis, or lymphoma, which has a similar magnetic resonance imaging (MRI) appearance. ${ }^{3}$

We present another case of solitary dural plasmacytoma with massive amyloid deposition and involvement of bone with soft tissue extension, which on 2 years of follow-up did not reveal any evidence of MM.

\section{Case Report}

In October 2015, a 25-year-old male patient presented with gradually and progressively enlarging painless scalp swelling over the left occipital region since 6 months. On examination the patient was fully conscious and oriented to time, place, and person. There was no cranial nerve deficit, and tone was normal. Power upper limb, shoulder (abduction/adduction), elbow (flexion/extension), grip: 5/5. Lower limb, hip (abduction/adduction), knee (flexion/extension), ankle (DF/PF): 5/5, DTR++. Planter $\mathrm{B} / \mathrm{L}$ flexor and no sensory system deficit was present. On investigation hemoglobin ( $\mathrm{Hb})$ was $13.2 \mathrm{~g} \%$, total leukocyte count (TLC) $10,660 / \mathrm{mm}^{3}$, differential leukocyte count (DLC) N68\%L20\%E06\%M06\%, blood urea $22 \mathrm{mg} \%$, and serum creatinine was $0.75 \mathrm{mg} \%$. Viral markers were negative, and coagulation profile was within normal range.

Magnetic resonance imaging of the brain revealed large well-defined extra-axial densely but heterogeneously enhancing soft tissue mass lesion in the left parieto-occipital region, which was destroying the left parietal bone, bulging into the scalp compressing the adjacent superior sagittal

C2019 Neurological Surgeons'

License terms

Society of India 
sinus without obvious invasion along with mass effect and midline shift to right side (-Fig. 1). The mass measured $8 \times 7.9 \times 6.8 \mathrm{~cm}$. No restricted diffusion or surrounding edema was seen. The findings were suggestive of a neoplastic lesion, and possibilities of atypical meningioma or sarcoma or vascular lesion were considered on radiology.

Left parieto-occipital craniotomy and gross total excision of tumor mass were done. Perioperatively tumor was pinkish, gray, highly vascular, and present extradurally involving and invading the calvarium with large extracranial extension. It was gradually dissected along the extradural plane. Dura was highly vascular. Broad dural origin was coagulated, and gross total decompression of tumor mass was done.

Biopsy received in the laboratory was large soft to firm, grayish brown, and tissue piece measuring $10 \times 9 \times 5 \mathrm{~cm}$ in size along with bony piece $3 \times 2 \times 1 \mathrm{~cm}$. On microscopy it revealed exuberant production of amorphous, eosinophilic, fibrillary material in form of small and large nodular aggregate. Interspersed with these amorphous deposits were dyscohesive tumor cells. The tumor cells were small round to oval cells with eccentric uniform nuclei, coarse chromatin, inconspicuous nucleoli, and thin rim of granular cytoplasm and characteristic "perinuclear hof," resembling plasma cells (-Fig. 2A, B). This abundant deposit was congophilic, and underpolarized microscopy showed apple green birefringence. On immunohistochemistry, the tumor cells were positive for CD138 with $\mathrm{k}$-light-chain restriction. Therefore, the diagnosis of plasmacytoma with amyloid deposition was made. Myeloma workup (serum and protein electrophoresis with immunofixation, skeletal survey, serum immunoglobulin, and free light-chain assay) was negative. The patient received radiotherapy of 46 Gy in 23 fractions to primary site. We followed up the patient to rule out progression to MM. The patient was free from systemic progression for the past 24 months although future course remains to be seen.

\section{Discussion}

There are only a few cases described in the literature of intracranial involvement of plasmacytoma without any evidence of MM. ${ }^{3-6}$ There are case reports of presentation of dural or cranial plasmacytoma at any time during the course of $\mathrm{MM}^{7,8}$ or even years after complete remission of MM. ${ }^{9}$

In our case, the patient was diagnosed with dural plasmacytoma spreading to the bone with soft tissue extension. Involvement of the dura mater arising from bony lesions in the calvaria, skull bone, and nasal or paranasal

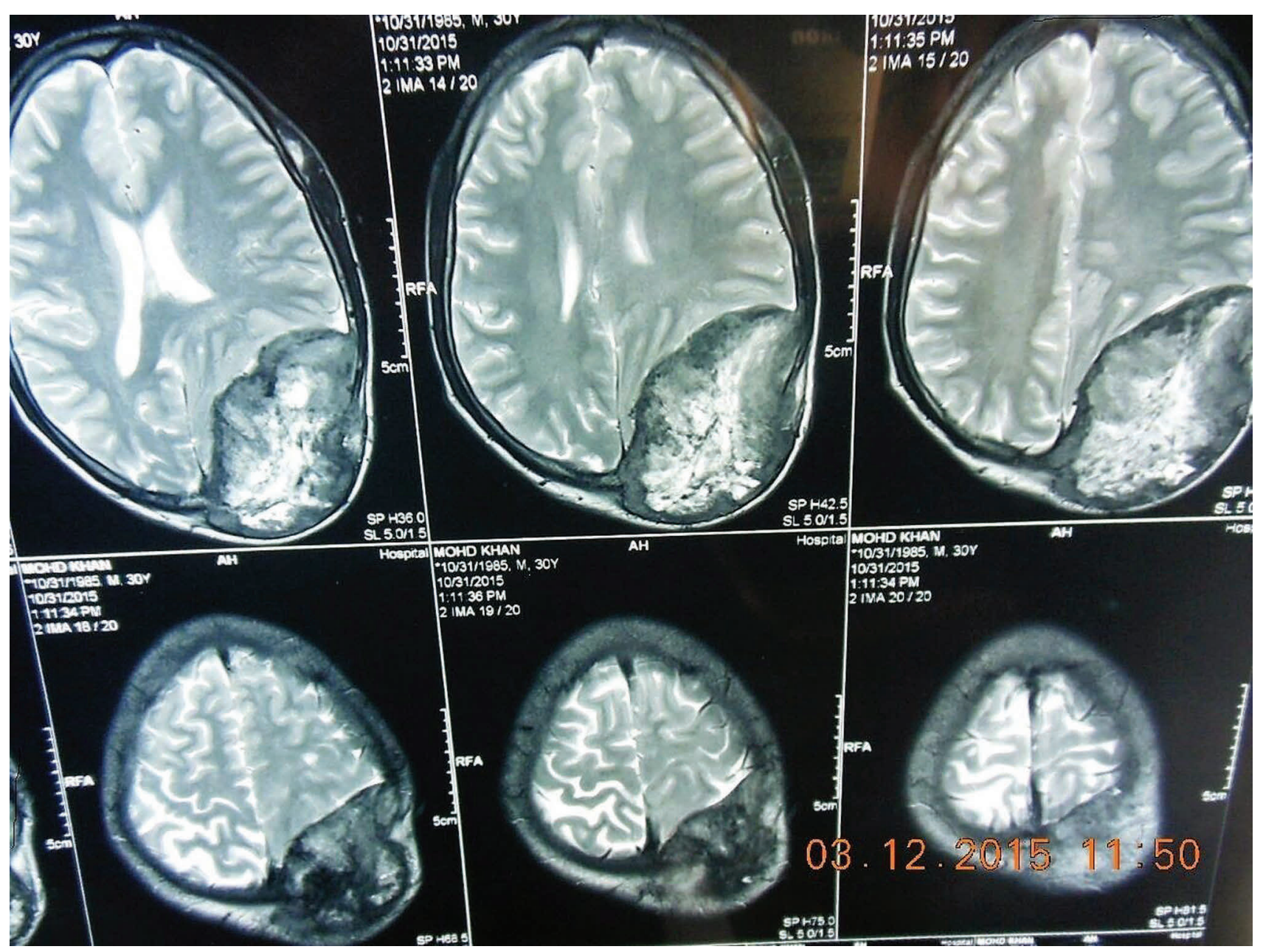

Fig. 1 MRI of the brain-gradient echo imaging showing dural tail, calcification, and bone destruction. 

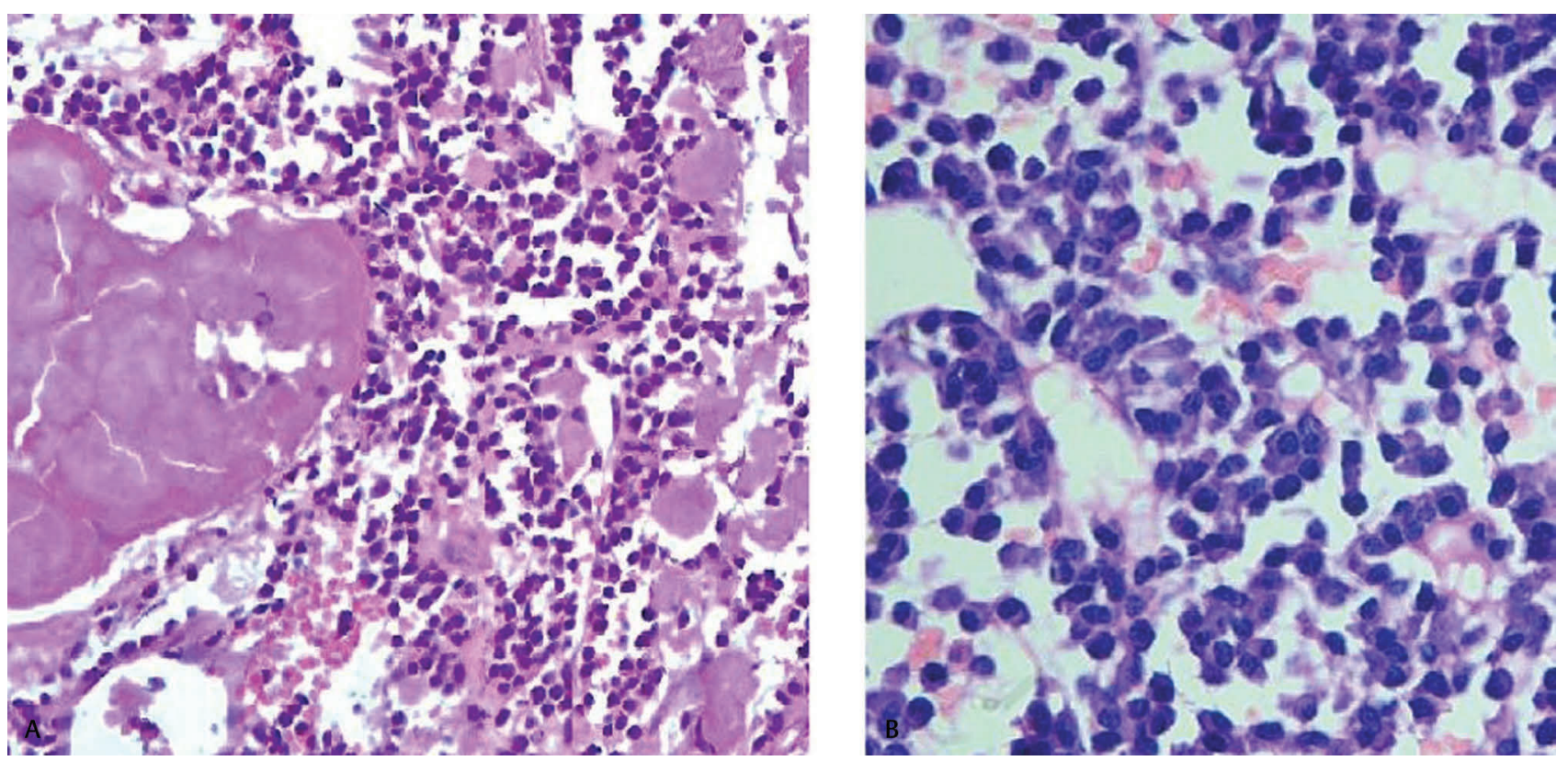

Fig. 2 (A) Histologic section showing amorphous eosinophilic deposits (amyloid) and dyscohesive plasma cells (H\&E, 20×). (B) Histologic section showing plasma cells (H\&E, 40×).

sinuses are described by Cerase et al. Dural involvement resulted from direct spread of contiguous bone lesion may be a possibility to be considered, because the vascular structure of the dura mater permits the extension of plasma cell tumors from primary contiguous bone to the subsequent subdural space and involvement of the dura. Another possibility is of primary dural lesion with infiltration, destruction of bone, and soft tissue extension. ${ }^{4,10}$ The second possibility is more appropriate in our case because of broad dural base with dural tail mimicking meningioma.

However, this differentiation is important because the percentage of progression to $\mathrm{MM}$ is significantly higher if it is primarily a bone plasmacytoma in comparison to extramedullary plasmacytoma. Risk for progression of bone plasmacytoma to myeloma is at a rate of 65 to $84 \%$ in 10 years and 65 to $100 \%$ in 15 years, and in spite of a curative treatment, the median time to progression to MM is 2 to 3 years. ${ }^{11}$ However, in a review of 721 extramedullary plasmacytoma cases by Alexiou et al, after treatment, approximately $65 \%$ of the patients had no recurrence and did not progress to MM, whereas $22 \%$ experienced recurrence and $15 \%$ of the cases evolved into MM. ${ }^{12}$ Therefore, in our case the future outcome is still uncertain.

Treated MM after few years can also develop into meningeal plasmacytoma. There is theory that this meningeal involvement may be present from the start of the disease, increasing its presence during development of this pathology. This is based on the fact that most treatments for myeloma do not cross the blood-brain barrier., ${ }^{8,13}$

There are no pathognomic radiologic features for dural plasmacytoma. They may be confused with other tumors as they are not remembered primarily in radiologic diagnosis.
The differential diagnosis includes meningioma, lymphoma, or metastasis.

On computed tomographic (CT) scan, lesion will show signal iso- or hyperintensity. An MRI shows T1 signal iso- or hyperintensity and marked T2 signal hypointensity. This presentation mimics the images of a meningioma that is the main differential diagnosis together with metastasis and lymphoma. ${ }^{4}$

Therefore, a histologic study is essential to confirm the diagnosis. For definitive diagnosis, systemic MM must be ruled out and monoclonal nature of tumor cells must also be confirmed to rule out inflammatory process.

Because of their rarity, the literature of management of cranial plasmacytoma is limited. At present, consensus agreed that the solitary plasmacytoma are to be managed by gross total resection with adjuvant radiation therapy because the tumor is very radiosensitive.,10 Tumor of the skull base is treated by subtotal or total resection followed by radiotherapy, or radiation alone. Systemic therapy is recommended for refractory and/or recurrent disease..$^{10,14}$

Systemic therapy is also helpful in cases in which the tumor is in proximity to vital structures such as optic chiasma, and attending physicians felt that the location of the tumor precluded therapy with radiotherapy or surgical intervention. ${ }^{12}$ Chemotherapy can also be administered for secretory tumors. ${ }^{5}$

\section{Conclusion}

Plasmacytoma should be considered in the differential diagnosis of any solitary dural mass in a known case of MM. In the absence of MM, this is a quite rare entity and it is 
difficult to predict the prognosis. Therefore, all patients need further follow-up to detect possible progression of MM.

\section{Source(s) of Support}

None.

\section{Conflict of Interest}

None.

\section{References}

1 Caers J, Paiva B, Zamagni E, et al. Diagnosis, treatment, and response assessment in solitary plasmacytoma: updated recommendations from a European Expert Panel. J Hematol Oncol 2018;11(1):10 https://www.ncbi.nlm.nih. gov/pmc/articles/PMC5771205/ [serial online]

2 Labib ML, Som PM. Unusual extramedullary plasmacytoma of the head and neck: a case series. Neurographics 2017;7(2):115-120

3 Azarpira N, Noshadi P, PakbazS, Torabineghad S, Rakei M, Safai A. Dural plasmacytoma mimicking meningioma. Turk Neurosurg 2014;24(3):403-405

4 Khalili RP, Mokhtari M, Fard SA, Neshat A, Norouzi R. Solitary dural plasmacytoma with parenchymal invasion. Asian J Neurosurg 2015;10(2):102-104

5 Hasturk AE, Basmaci M, Erten F, Cesur N, Yilmaz ER, Kertmen H. Solitary dural plasmacytoma mimicking meningioma and invading calvarium. J Craniofac Surg 2013;24(2):e175-e177
6 Webb M, Barrett C, Barrett S, van Rensburg JJ, Louw V. Cranial plasmacytoma: a case series and review of the literature. Indian J Hematol Blood Transfus 2013;29(1):43-47

7 Sahin F, Saydam G, Ertan Y, Calli C, Dönmez A, Tombuloglu M. Dural plasmacytoma mimicking meningioma in a patient with multiple myeloma. J Clin Neurosci 2006;13(2):259-261

8 Laribi K, Mellerio C, Baugier A, et al. Meningeal involvement in multiple myeloma. Clin Case Rep 2015;3(2):84-87

9 Morgenstern P, Pisapia D, Ramakrishna R. Calvarial plasmacytoma mimicking meningioma as the initial presentation of multiple myeloma. Cureus 2017;9(3):e1126 https://www.ncbi.nlm.nih.gov/pmc/articles/PMC540981

10 Cerase A, Tarantino A, Gozzetti A, et al. Intracranial involvement in plasmacytomas and multiple myeloma: a pictorial essay. Neuroradiology 2008;50(8):665-674

11 Bolek TW, Marcus RB Jr, Mendenhall NP. Solitary plasmacytoma of bone and soft tissue. Int J Radiat Oncol Biol Phys 1996;36(2):329-333

12 Alexiou C, Kau RJ, Dietzfelbinger $\mathrm{H}$, et al. Extramedullary plasmacytoma: tumor occurrence and therapeutic concepts. Cancer 1999;85(11):2305-2314

13 Gascón N, Pérez-Montero H, Guardado S, D’Ambrosi R, Cabeza MA, Pérez-Regadera JF. Dural plasmacytoma with meningeal myelomatosis in a patient with multiple myeloma. Case Rep Hematol 2018;2018:6730567 https://www.hindawi.com/ journals/crihem/2018/6730567/abs

14 Terada T. Multiple myeloma presenting as an intracranial plasmacytoma: a case report. Cases J 2009;2:9110 https:// www.ncbi.nlm.nih.gov/pmc/articles/PMC2803907/ 